\title{
Description et définition des diverses figures de cavitation observées dans les machines et conduits hydrauliques
}

\author{
PAR J. BEAUFRĖRE, \\ INGÉNIEUR AU DËPARTEMENT «ESSAI 》 \\ DU SERVICE « LABORATOME ET FSSAIS HYDRAULIQUES D'E.D.F., CHATOU
}

(Texte résumé, le texte intégral devant être publié ultérieurement)

INTRODUCTION

Le sujet de ce mémoire a été donné par la Commission de cavitation de la Societé Hydrotedmique de France à un groupe de travail composé de MM. BEauFRÈre, ingénieur au Département Essais, Electricité de France, rapporteur; Huciennin, professeur à l'Ecole Nationale d'Ingénieurs des Arts et Métiers; Menez, ingénieur du Génie Maritime en service au Bassin des Carènes; Rata, ingénieur au Département Essais, Electricité de France.

Nous donnons ici des extraits du rapport de ce groupe de travail, rapport qui paraîtra ultérieurement in extenso dans La Honille Blanche.
Ce rapport vise a définir un langage commun permettant de décrire sans ambiguïté les différents types de cavitation que l'on peut lencontrer de manière à normaliser les appellations qui leur sont généralement données. Il est souhaitable que les notations proposées soient utilisées par les spécialistes de la cavitation, mais il est aussi indispensable que ceux-ci fassent connaitre leurs observations s'ils rencontrent des cas qui puissent difficilement être identifiés en vue d'améliorer et de généraliser la méthode de classification utilisée.

\section{1. - MÉTHOdES D'OBSERVATION DE LA CAVITATION}

\begin{abstract}
L'observation des différentes figures de cavitation en vue de leur définition et de leur description se fait par. voie optique, et les différents moyens utilisés sont : l'œil, l'appareil photographique ou cinématographique, la cinématographie ultra-rapide et la télévision. Tous ces moyens doivent être utilisés avec un éclairage approprié, qui dépend de la figure à observer (en particulier de la rapidité de ses fluctuations) et de la vue que l'on
\end{abstract}

veut avoir du phénomène. Si l'on veut avoir une vue d'ensemble, on opère avec un éclairage de durée relativement longue ou avec un éclairage fixe et une ouverture lente (ex. fig. 11), et si l'on yeut avoir une vue nette de la figure de cavitation et de sa composition, on opère avec un éclairage très court (éclair d'un flash rapide ou d'un stroboscope) (ex. fig. 12). 


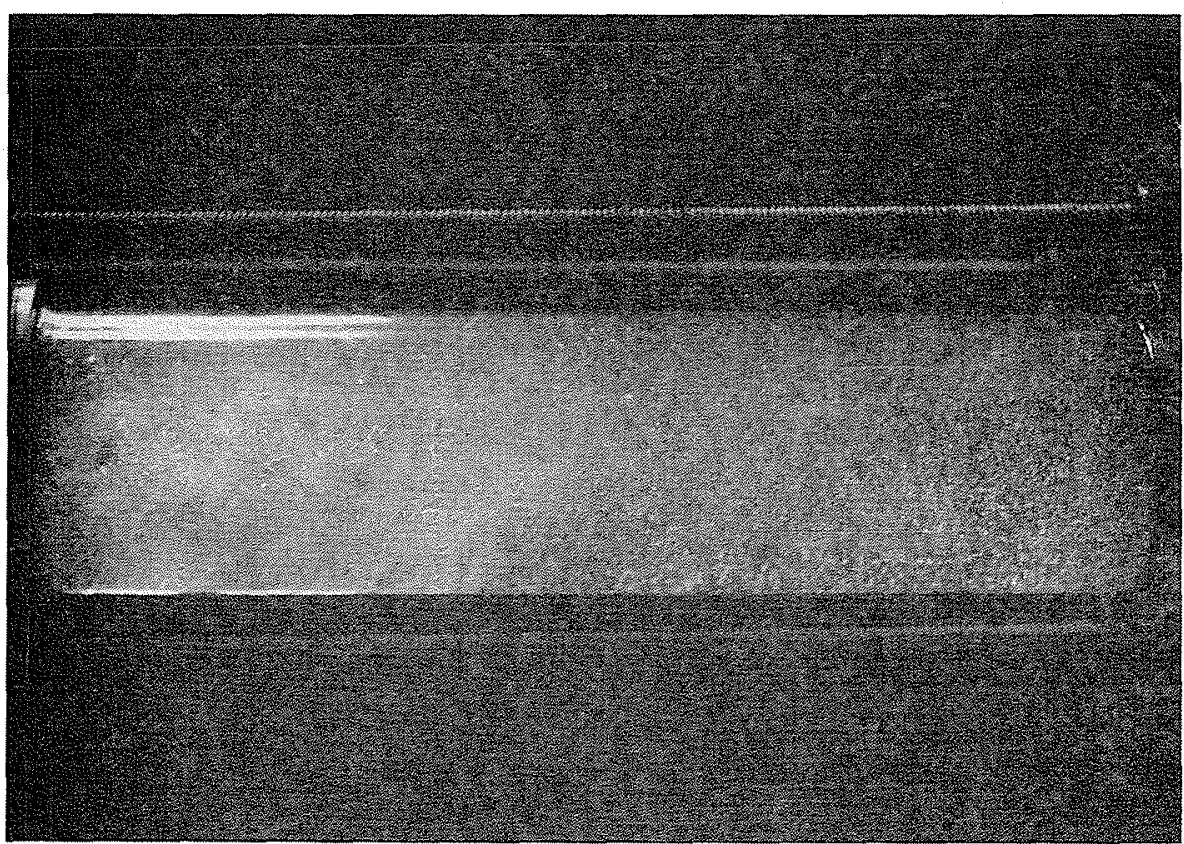

Fig. 1

Diaphragme.

Cavitation 511 et 411 .

\section{2. - CRITĚRES DESCRIPTIFS DE LA CAVITATION}

Les critères retenus pour définir et decrire les diverses figures de cavitation sont au nombre de trois : aspect, localisation et stabilité.

2.1. L'expérience montre que les aspects différents présentés par la cavitation peuvent être groupés en un nombre relativement réduit de eatégories: les poches, les bulles, les lames et les tourbillons.

2.1.1. Jes dimensions des poches sont de l'ordre de celles de l'installation où elles apparaissent. Elles se rencontrent seules ou en nombre limité.

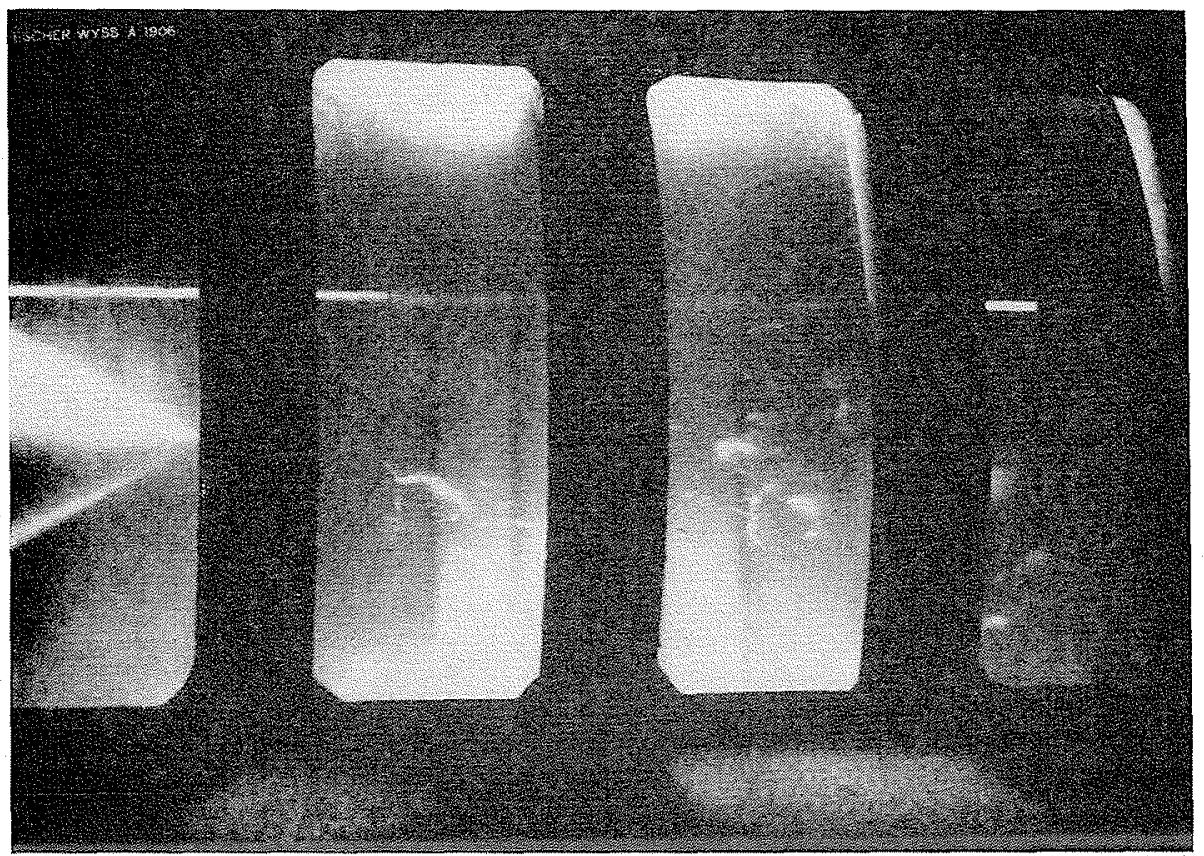

FIG. 2

Déchargeur.

Cavitation 917 
2.1.2. Un aspect très courant de la cavitation est la cavitation par bulles. Les dimensions des bulles sont petites vis-à-vis de celles de l'installation; elles sont grossièrement sphériques. Leur taille, leur nombre et leur arrangement amène à distinguer: les bulles isolées, les bulles en grappes et les bulles en nuages. Les bulles en grappes sont en nombre relativement grand, elles peuvent ètre toutes distinguées les unes des autres, et étudiées individuellement. Les bulles en nuages sont de dimensions très petites et en très grand nombre, on ne peut les étudier séparément.

2.1.3. Les lames sont des poches dont une dimension est très faible vis-à-vis des deux autres.

2.1.4. Le tourbillon cavitant consiste en une cavité ou un ensemble de cavités dont une dimension est très grande vis-a-vis des deux autres. Les tourbillons cnvitants peuvent se ranger en deux classes : les tourbillons de grandes dimensions, à l'aspect de torsades brillantes ou de torches et les tourbillons de très petites dimensions, semblables a des filaments fulgurants.

2.2. Le critère descriptif des figures de cavitation qui consiste à étudier ces diverses figures d'après le lieu oì elles apparaissent est aussi très naturel. Il met en évidence trois classes simples de figures : celles qui apparaissent au sein d'un écoulement, celles qui apparaissent au voisinage d'une surface et eelles qui sont accrochées à une surface. Cette derniere classe peut se diviser en deux : cavitation accrochée à une surface et se terminant à l'infini (au sein de l'écoulement) et cavitation accrochée a une surface, se terminant sur une surface.

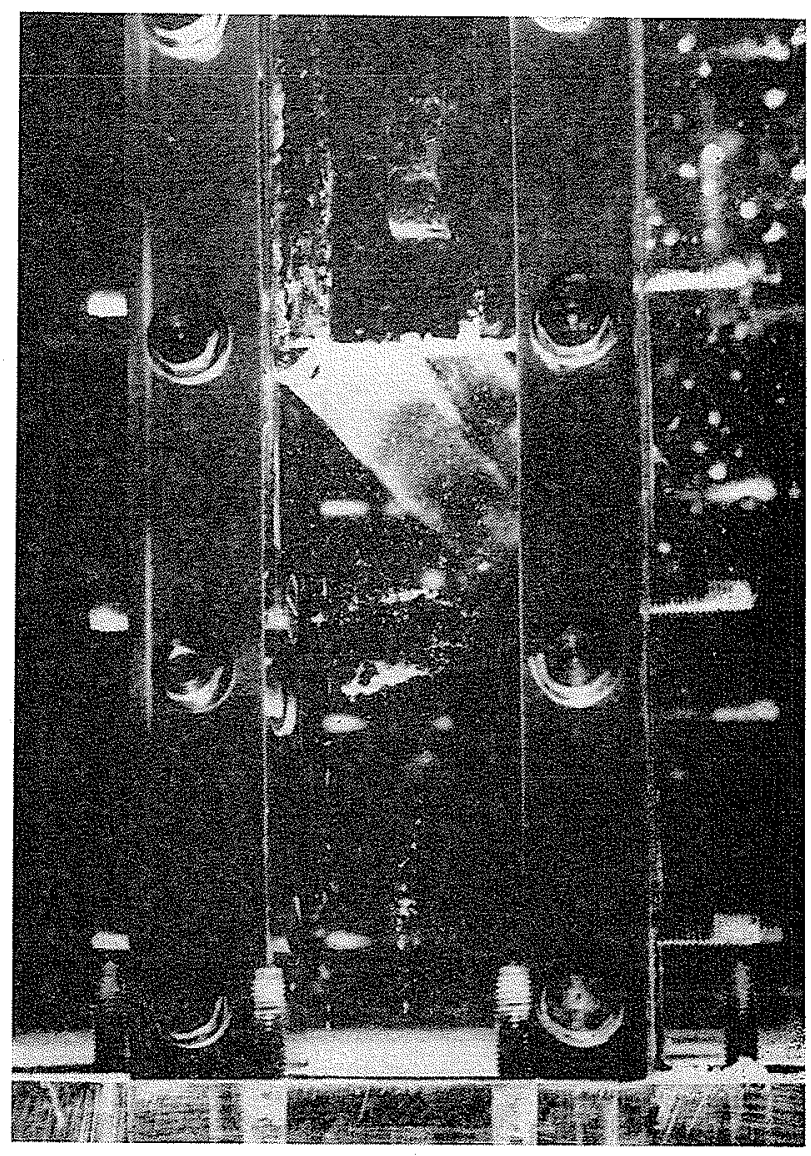

Fic. 3

Vamne. Cavitation 411 et 511 .
FI: 4

Cavitation principale 641 . Turbine supercavitante.

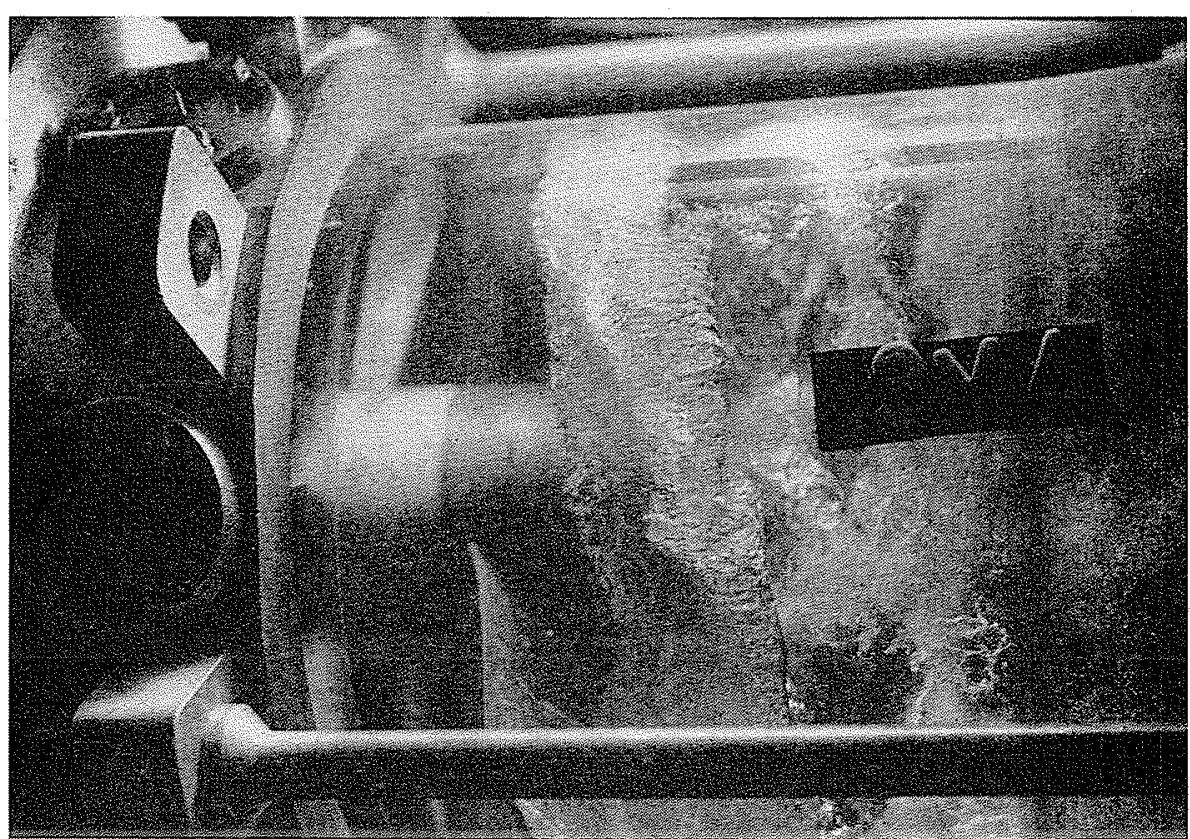




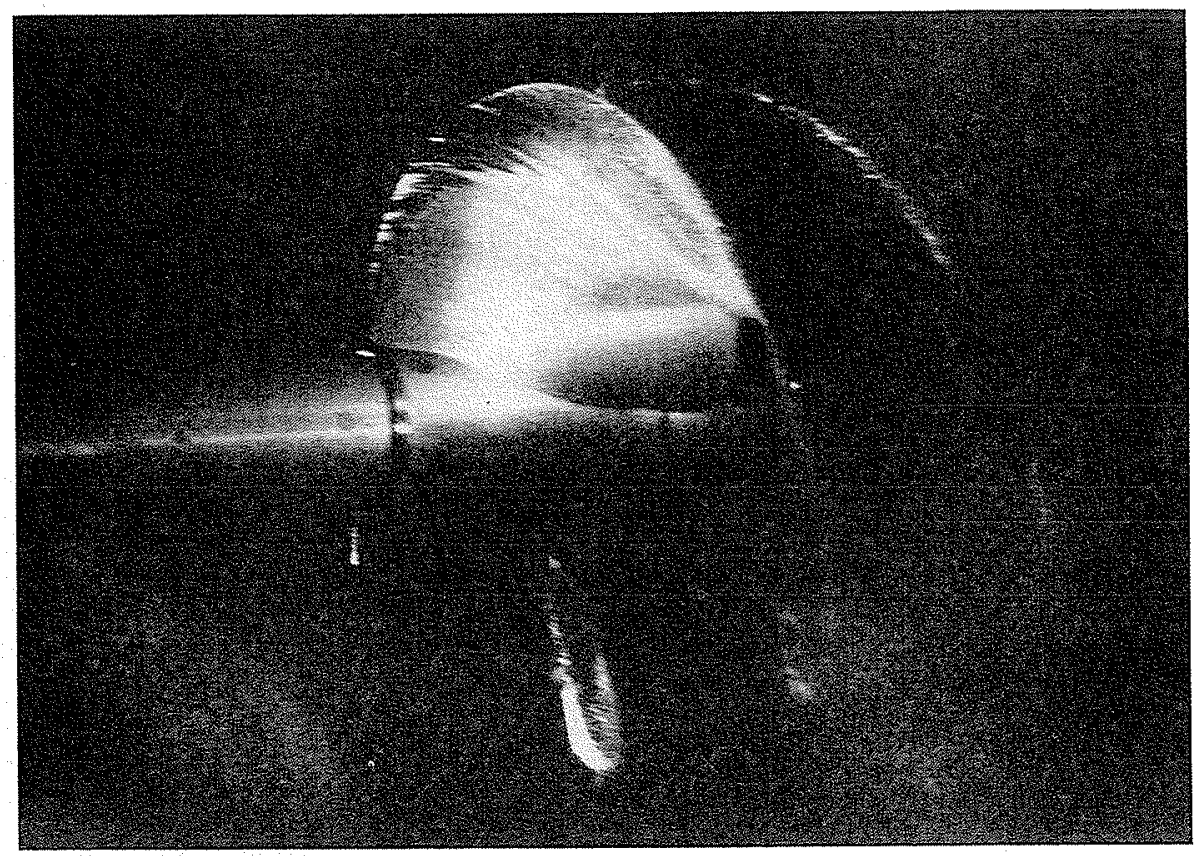

FiG. 5

Hélice marine.

Cavitations 631 et 741

combinées.

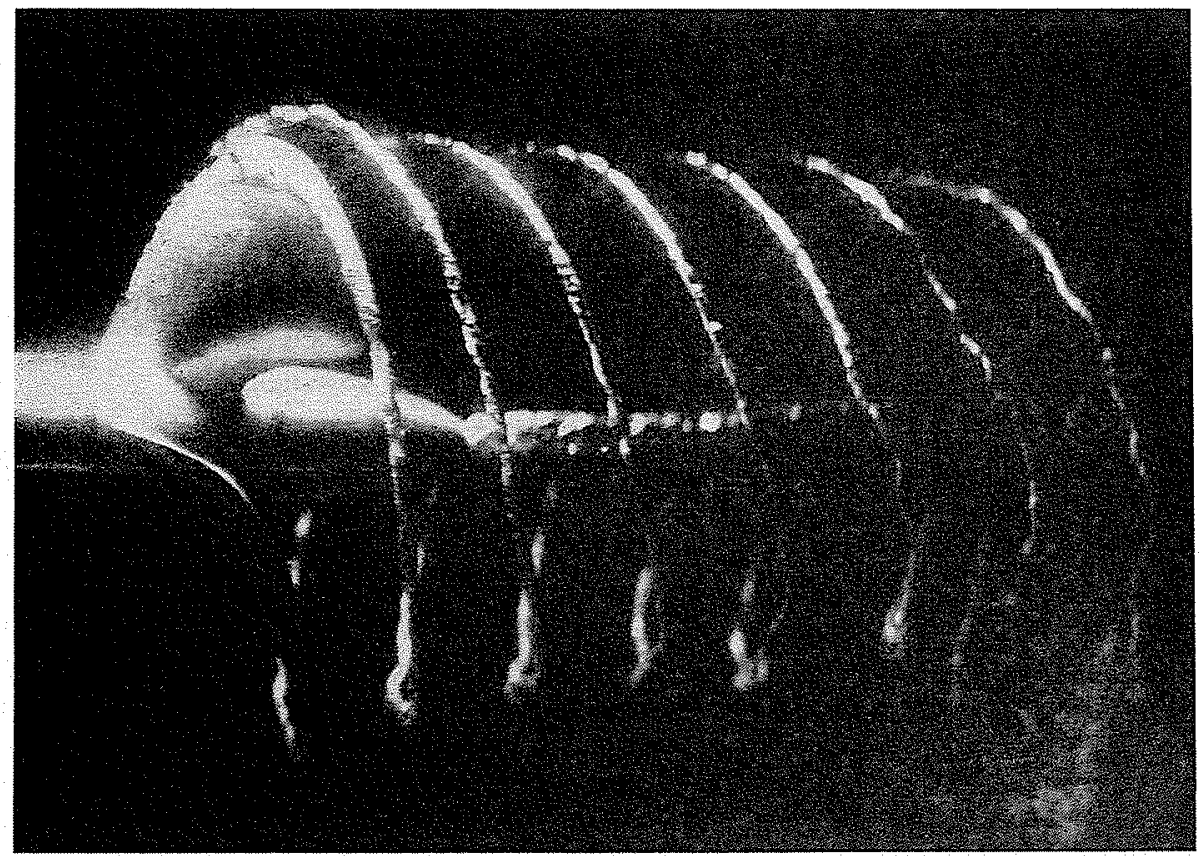

Fig. 6

Hélice marine.

Cavitation 741 et 841 . 


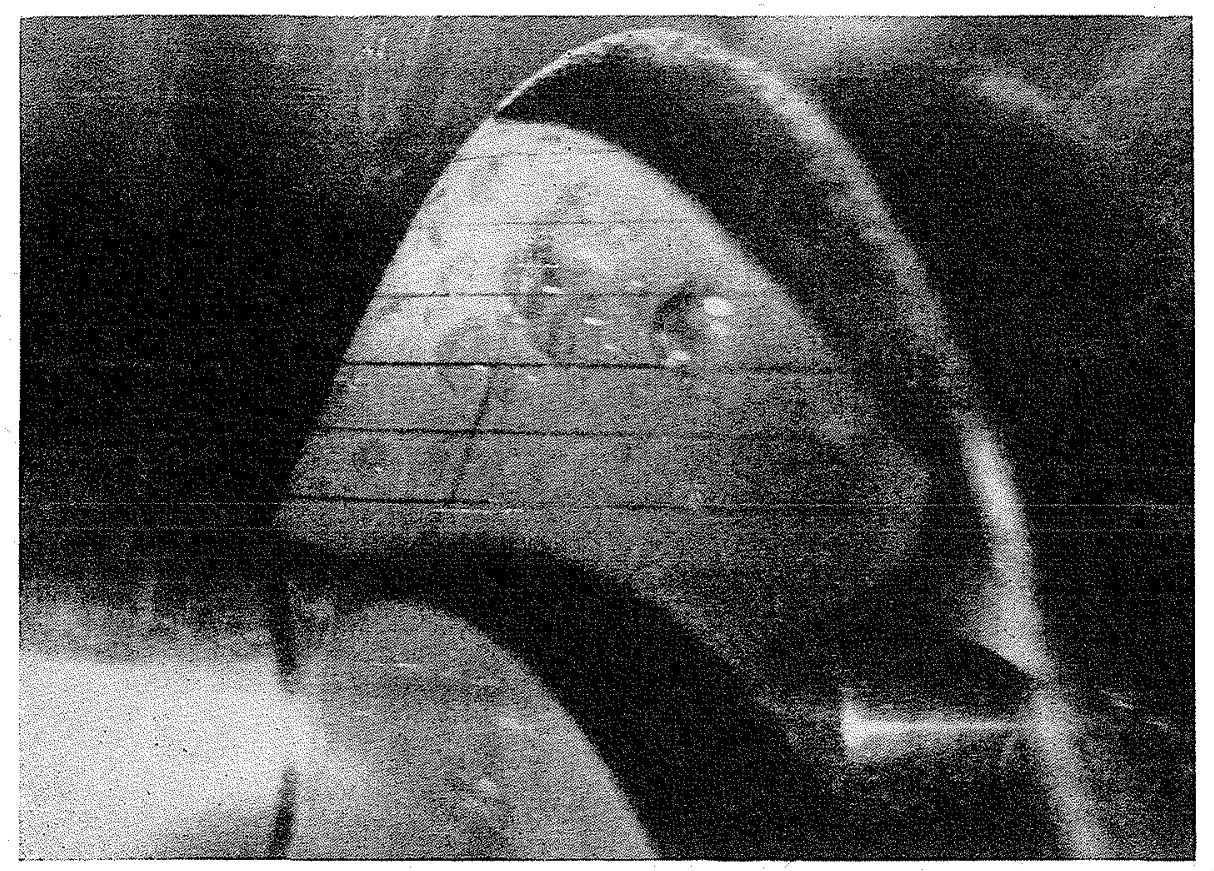

Fig. 7

Hélice marine.

Cavitation 325 et 641 .

Fic. 8

Pompe hẻlice.

Cavitations 631 ,

912 et 417 combinées.

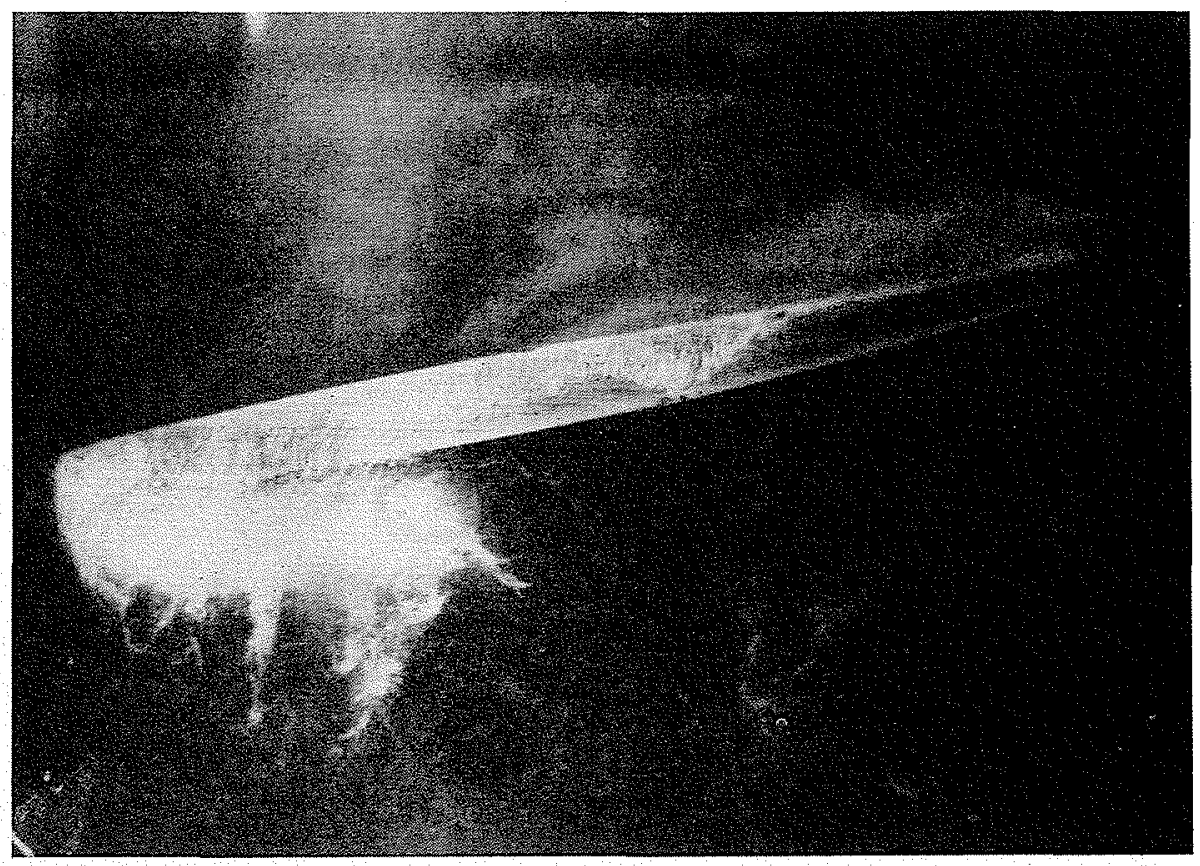




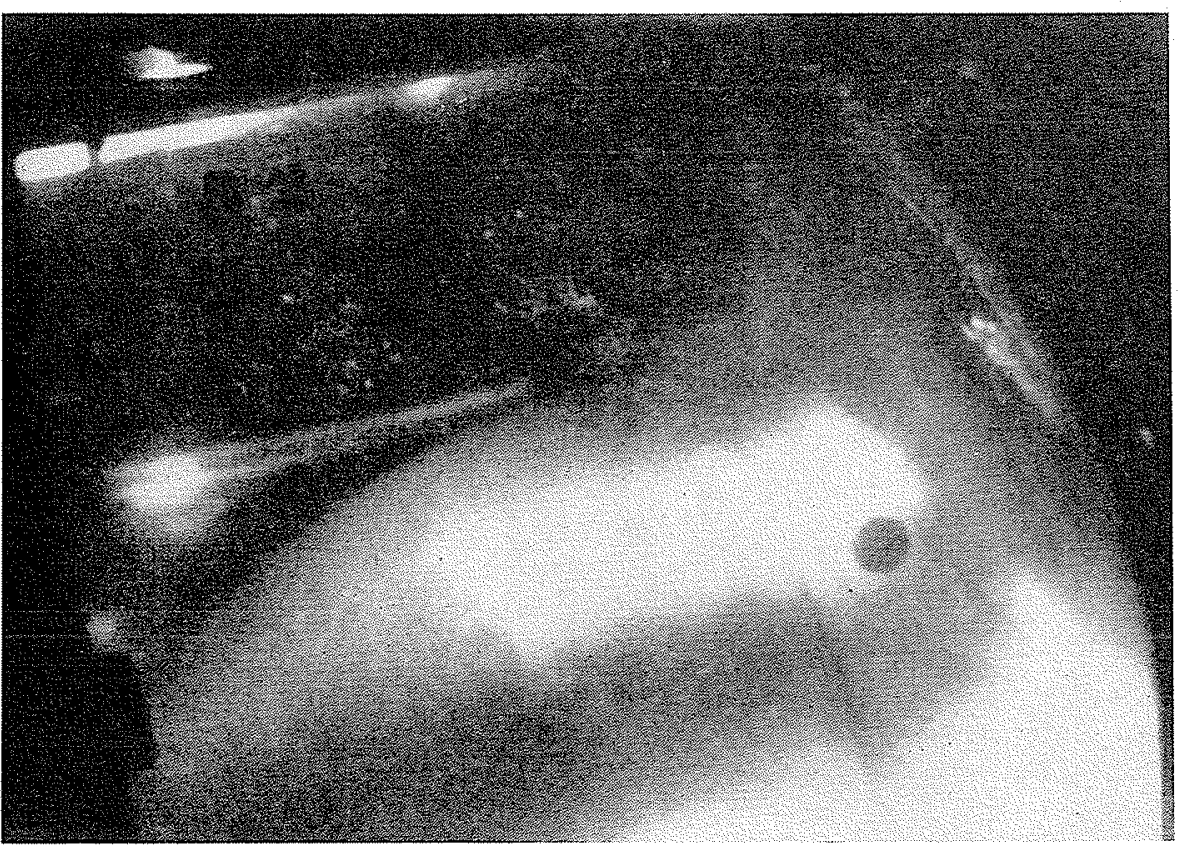

FiG. 9

Turbine Kaplan.

Cavitation 232, 631 et 411 .
2.3. La stabilité d'une figure de cavitation s'étudie dans le temps et dans l'espace. Au sens strict du terme, toutes les figures de cavitation sont instables, car, en un lieu donné, la cavitation est un phénomène qui évolue en général très rapidement dans le temps, et ce lieu peut lui-même varier. On peut cependant distinguer les figures «stables en moyennes» des figures instables: une figure stable en moyenne est une figure dont les déformations sont très rapides dans le temps, mais très petites devant les dimensions de cette figure, de telle sorte que les fronicies de la cavité varient peu. Plus séneralement, on appellera figure stable une fignre qui, observée par un moyen bien détermine se pressnte toujours de la même façon, quel que soit linstant de l'observation.

Linstabilité des figures doit être caractérisée par la nature des variations dans le temps (périodiques ou aléatoires, mides ou lentes) et dans l'espace.

2.4. Les trois criteres énumérés permettent de définir des éléments simples de la cavitation. En réalité, il arive souvent que l'on observe plusieurs de ces éléments simples, combines ou non, d'importance différente, ce qui amène à distinguer : les figures principales, les figures combinées et les figures secondaires.

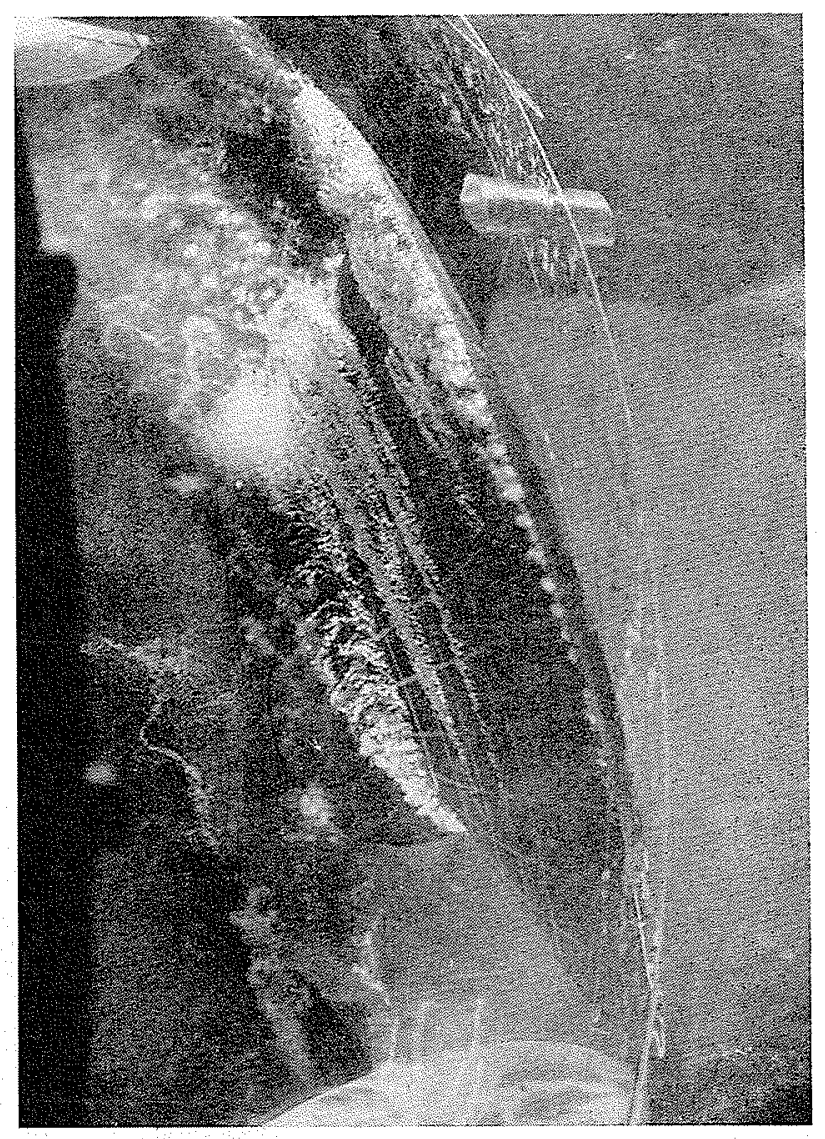

FIG. 10

Turbine Kaplan.

Cavitation $421,325,131,511$ et 917 


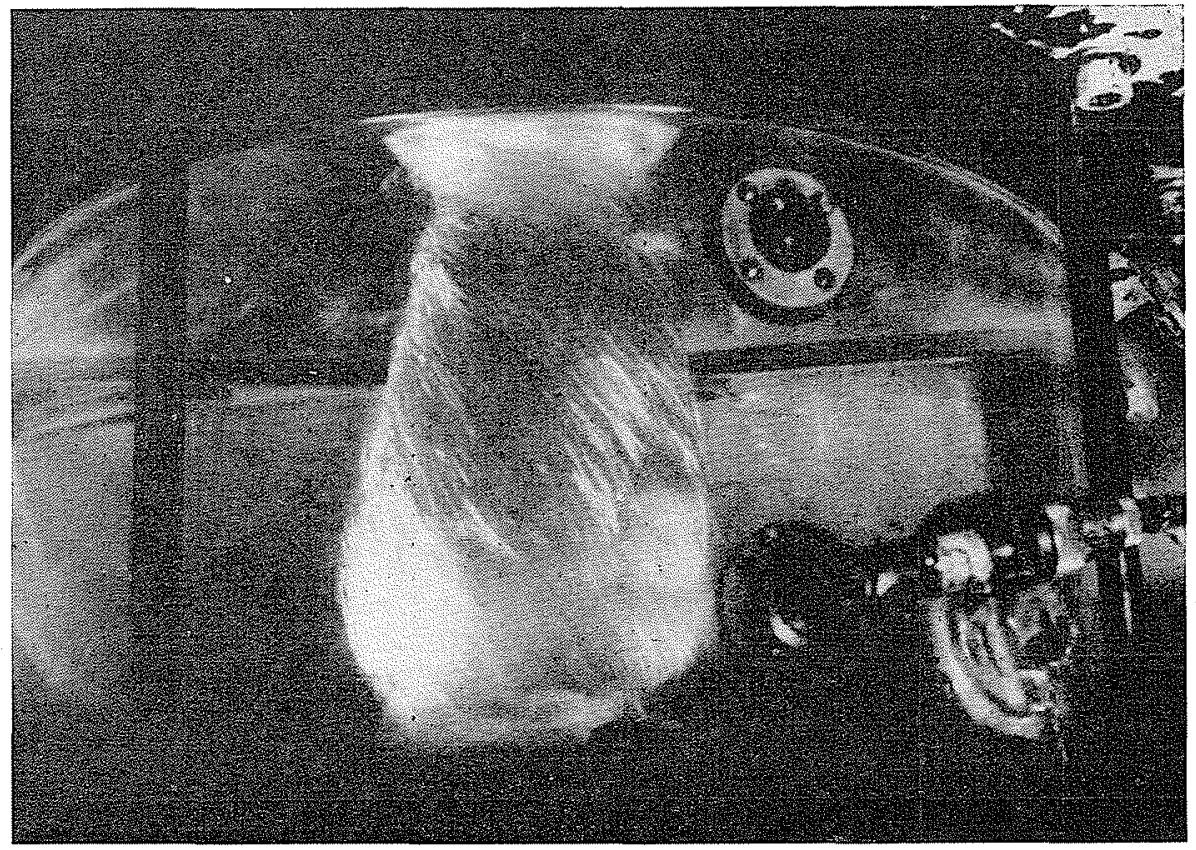

Fig. 11

Turbine Francis.

Cavitation 841.

Temps de prise de vue :

$1 / 100^{\prime \prime}$.

\section{3. - CONCLUSION - NORMALISATION}

L'utilisation des trois critères descriptifs permet de classer de façon relativement précise toutes les figures de eavitation que l'on peut rencontrer dans les machines et conduits hydrauliques. Nous domons dans le tableau ci-dessous la liste des termes qui ont été retenus pour la normalisation, ainsi que les symboles qui permettent

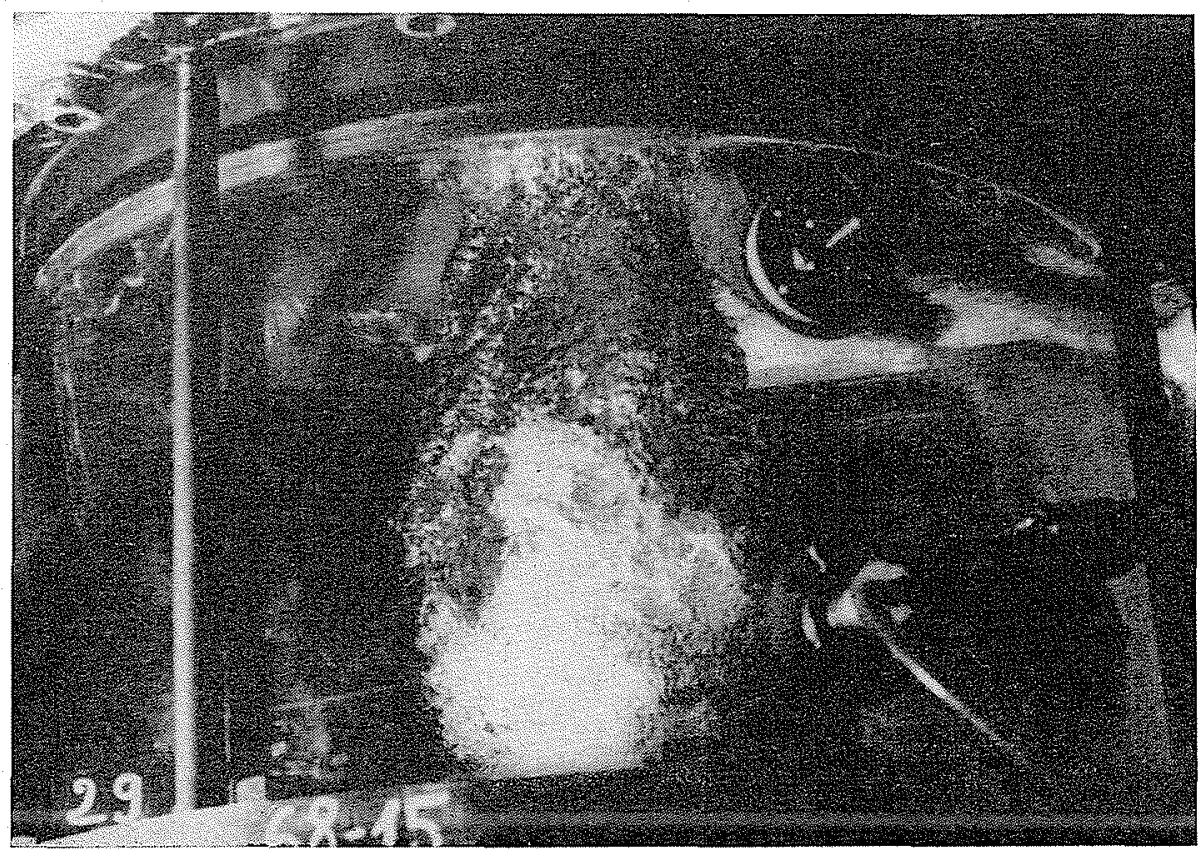

FIG. 12

Même sujet que figure 11.

Temps de prise de vue : $1 / 50000^{\circ}$. 
de représenter chaque figure de cavitation par un groupe de trois chiffres, le premier étant relatif ì l'aspect, le deuxième à la localisation et le troisième à la stabilité. De plus, il faut bien préciser, pour chaque figure décrite, si e'est un aspect principal ou un aspect secondaire de la cavitation, ou si les figures principales observées sont séparées ou combinées.

\begin{tabular}{|c|c|c|}
\hline CRITERE & APPELATTOON & Sy MBBOLE \\
\hline ASPECT . & 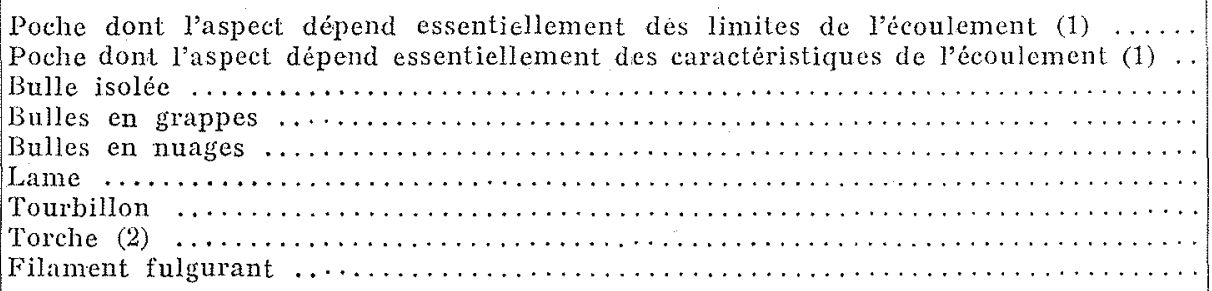 & $\begin{array}{l}1 \\
2 \\
3 \\
4 \\
5 \\
6 \\
7 \\
8 \\
9\end{array}$ \\
\hline LOCALISATION. & 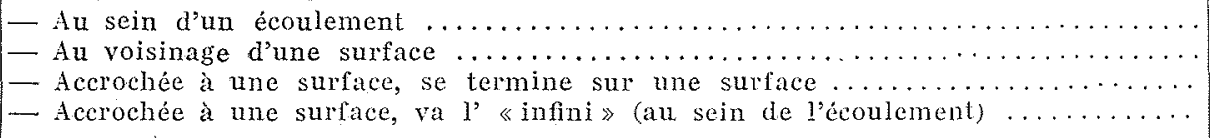 & $\begin{array}{l}1 \\
2 \\
3 \\
4\end{array}$ \\
\hline STAUBILITE: & 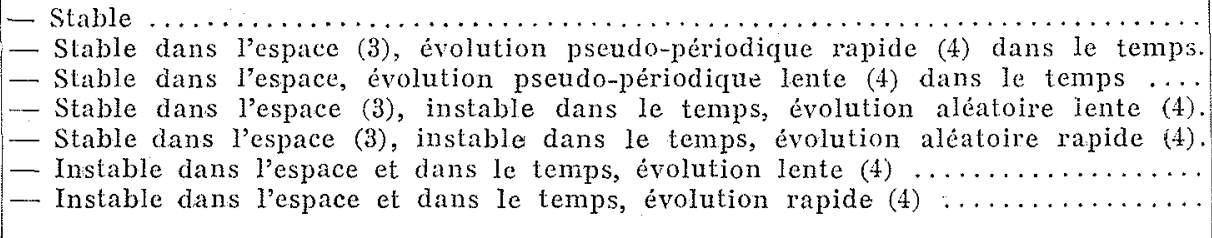 & $\begin{array}{l}1 \\
2 \\
3 \\
4 \\
5 \\
6 \\
7\end{array}$ \\
\hline
\end{tabular}

(1) Nous avons donné deux numéros à l'aspect en poche de la cavitation pour pouvoir distinguer deux types assez différents :

- Les poches dont l'aspect dépend essentiellement des limites de l'écoulement sont les poches quí remplissent une partie du conduit (coup de bélicr) ou bien sont accrochées ̀̀ une singularité de ce conduit (sillage d'une aspérité ou d'un obstacle non profilé);

- Les poches dont l'aspect dépend essentiellement des caractéristiques de l'écoulement sont les poches qui dépendent au premier chef du champ de pression dans l'écoulement, qui lui-même dépend des limites de l'écoulement. Mais nous considérons que l'effet de ces limites n'est pas direct, et qu'il cst beaucoup moins sensible que dans le premier cas. (Exemple : poche pseudo-périodique sur l'extrados d'une pale de turbomachine.)

(2) Le terme de torche est réservé au tourbillon qui apparaît sur les ogives des machines tournantes.

(3) Par stable dans l'espace, nous entendons : figure de cavitation qui se déforme en un lieu donné, ou se produit toujours de façon pseudo-périodique at aléatoire, au même endroit.

(4) Par rapide ou lent, nous entendons un phénomène dont les vitesses de variations sont à l'échelle de la milliseconde ou de la seconde.

\section{DIS C US SION}

président: M. BERGERoN

M. le Président remercie tout particulièrement M. Beaukríre, ainsi que les membres du groupe qu'il a constitué, pour leur rapport précis, présenté avec une grande clarté et comportant des propositions ariginales.

Il informe le Comité qu'au sein de la Commission pour l'étude de la cavitation, la classification des figures de cavitation en quatre catégories : poches, bulles, lames et tourbillons, n'a saulevé aucune critique. Par contre, il y a eu discussion à propos des trois criteres : aspect, localisation et stabilité, ainsi que sur les notations à adopter pour caractériser, chaque flgure, soit avec des lettres, soit avec des chimes.

M. le Président souligne l'importance de ce rapport, qui constitue non seulement un élément de base appelé a permettre d'adopter un langage commun entre spécialistes, mais aussi le point de départ de travaux ultéricurs. C'est ainsi que le groupe de recherche constitué pour l'étude de l'infuence des diverses figures de cavitation sur les bruits, érosions et écoulements va être le premier utilisateur de la classification et des criteres proposés par M. Beavraìn, puisque le but de ce nouveau. groupe est justement d'essayer de préciser les effets des différentes ligures de cavitation.

Pour ces différentes raisons, il serait très désirable que te plus grand nombre de personnes expriment lear opinion.

M. le Président ouvre alors la discussion.

M. Browel souligne l'intérêt présenté par le rapport du groupe de travail de M. Bravrrére, qui constitue probablement la première tentative des hydrauliciens de toutes origines pour parler un langage commun.

Il craint cependant que la homenclature propose soit trop compliquée pour devenir d'usage courant. Il pense, par exemple, qu'il est inutile d'introduire deux types de poches, alors qu"un seul suffirait et que, de meme, le terme "tourbillon" et le terme " torche" correspondent à un même phénomexe, hien que ne se présentant pas aux mêmes endroits. M. Bryder. pense egalement qu'il vaudrait mieux, pour la localisation, dire " ku sein de l'écoulement " plutôt qu" "à l'infini".

Mais il lui semble surtout que le troisieme critère devrait comporter moins de rubriques; compte tenu de ce qu'il ne 
s'agit pas en fait de stabilité au sens strict du terme, M. Bropr. propose, pour troisième critère, la nomenclature suivante :

\section{- cavitation permanente,}

- cavitation périodique ou pseudo-périodique,

- cavitation à variations aléatoires.

M. Brom. insiste enfin sur l'importance des moyens d'olse:vation dans la façon même de caractériser une cavitation donnée : en éclairage continu par exemple, une grappe p.ut très bien être qualifiée de nuage et un écoulement à variations periodiques rapides qualifié de permanent. Mais on alourdirait probablement inutilement la nomenclature en ajoutant un quatrième chiffre aux trois proposés $\left(^{*}\right)$.

M. Benufrème répond aux observations de M. Binder :

- Pour ce qui est des poches, il est apparu nécessaire de faire une distinction entre les deux types de poches. Les poches qui dépendent vraiment des conditions aux limites de l'écoulement sont celles qui apparaissent dans un coup de belicr ou ment sont celles qui apparaissent dans un coup de belier ou géométrique des limites de l'écoulement qui impose celle de la poche, quelles que soient les conditions de l'écoulement. Les autres poches dépendert au deuxième dergé des farmes de la surface, mais elles dépendent essentiellement des conditions de l'écoulement sur le point de la surface.

- Il est probable qu'il n'y aura jamais d'appareil d'observation suffisamment puissant pour observer les nuages comme on observe les grappes. La distinction entre grappes et muages repose essentiellement sur la densité des bulles. Si l'on a un nuage très dense de bulles, il sera impossible d'aller observer, derrière le rideau de petites bulles, l'évolution des autres bulles. $\left(^{* *}\right)$ et $\left({ }^{* * *}\right)$.

- On a donné un numéro différent aux tourbillons et aux torches, car celles-ci ont un aspect particulier. D'ailleurs, les tourbillons sont surtout abservés par les constructeurs d'hilices et il n'y a aucun inconvénient à ce que ces deux aspects aient le même numéro, le critère de localisation ne permet pas toutefois de distinguer les tourbillons des torches.

- Il est exact que le mot "stabilité " est imparfait. La définition de la stabilité devrait être telle que, si l'on imprime à une figure une petite variation élémentaire, cette figure de subisse qu'une variation élémentaire du même ordre. Il y aurait instabilité si la figure grossissait indéfiniment pour une aurait instabilité si la figure grossissait indéfiniment pour une
variation élémentaire, soit des champs de pression, soit des variation élémentaire, soit des champs de pression, soit des
champs de vitesse. Il vaut mieux retenir le critère de pernanence. Enfin, il est nécessaire, pour caractériser les genres d'évolution (pseudo-périodiques ou périodiques, aléatoires) de préciser si I'évolution est lente ou rapide.
M. Duport comprend bien l'hésitation de M. BrNDEL au sujet des définitions assez précises proposes par M. BEAUFrìne, Il est évident que dans certains cas d'expérience ou de mesure, on pourra hésiter entre 1 et 2 ou entre les diverses acceptions possibles concernant les notions de stabilité ou de permanence. S'il y a un doute, on peut toujours dire que c'est "l'mn ou l'autre". Mais il vaut micux essayer d'aroir un langage aussi précis et complet que possible.

D'autre part, M. Duron'r pense que ce n'est pas uniquement une question d'observation qui fait distinguer les grappes du muage. On peut penser que, dans une grappe, les bulles sont moins nombreuses at de dimensions plus importantes que dans un nuage, et que, dans un nuare, les bulles ne sont pas encore individualisees : une bulle peut donner naisance à plusieurs, par fragmentation, ou au contraire, plusieurs petites peuvent se rassembler en une plus grande. Mais ceci n'est qu'une hypothèse. On peut proposer enfin, en complement du rapport de M. BeAcrétre, d'utiliser par exemple le chifrre 0 pour laisser entendre que l'on hésite entre 4 et 5 , entre 1 et 2 , etc.

M. Hug rappelle l'un des points de vue initiaux de cette étude. Il est très difficile en efret de faire l'unaminité a priori sur une telle nomenclature et e'est seulement l'expérience pratique qui permet de faire les discriminations nécessaires. Il faut donc essayer cette nomenclature et si, dans un certain délai, deux ans par exemple, on s'aperçoit que l'on a trop précisé un point ou pas assez un autre, rien n'empèche d'y revenir avec, cette fois, la sanction de l'expérience.

M. le Président trouve la remarque de $M$. Hug très justifie et exprime les conclusions suivantes :

- Il sera vraisemblablement nécessaire de maintenir la notion d'évolution lente et rapide (demandée par M. Huguens qui doit avoir une répercussion sur l’intensité du bruit.

- S'il y a des discriminations surabondantes, elles seront éliminées par la pratique.

- Le rapport tel qu'il est présenté peut être considéré comme déjà utilisable, sinon définitif.

- Il y a lieu de remercier M. Binder des interventions qu'il a formulées tant aujourd'hui qu'au cours des réunions de Ja Commission de la Cavitation, interventions pertinentes dont il a toujours été tenu compte. On peut noter avec plaisir que les remarques de M. Brnder ne portent plus aujourd'hui que sur des précisions dans les définitions et non sur les principes de base.

M. Ie Président remercie à nouveau M. Beaverìne et les personnes ayant pris part à la discussion.
Les astérisques sont relatifs à des observations et réponses faites postérieurement a la discussion du 15 mars 1962.

(*) M. Brome indique que la nomenclature (critère 3 ) fait état de la "rapidité " ou de la "lenteur" des évolutions de la cavitation, mais ces notions demanderaient à être précisées. Il semble que le mieux serait de se référex à un "temps propre ", qui pourrait être de l'ordre de grandeur du temps mis par une particule fluide a parcourir l'obstacle cavitant. Dans le cas d'une machine, on pourrait prendre, par exemple, le temps standard égal à $1 / n$ ( $n$, nombre de tours de la machine par unité de temps); on a $D / V$ (D diamètre, $V$ vitesse) s'il s'agit d'une "machine" non tournante (vanne par exemple). On pourrait alors dire que l'évolution est lente si sa période moyenne était largement inférieure au temps propre; avec ce critere, les évalutions rapides seraient d'ailleurs probablement sous la seule dépendance de la turbulence.

$\left({ }^{*}\right)$ M. Beaufréne pense qu'une grappe et un nuage de bulles peuvent effectivement avoir le même aspect en eclairage continu, mais craint qu'en éclairage continu, toutes les figures de cavitation présentent le même aspect de brouillard flou. Ce qui distingue les nuages des grappes c'est, sur des observations nettes et non floues, l'aspect laiteux et l'aspect granuleux. La transition entre nuage et grappe est cependant continue.

$\left({ }^{* *}\right)$ M. DEGrors utilise couramment la distinction entre les bulles en nuages et les bulles en grappes dans la description de la cavitation par ultrasons. 